\title{
25 Research Square

\section{A Method For The Quantitation Of The Metabolism Of Soluble Amyloid Precursor Protein Isoforms Using A Stable Isotope Labeling Kinetics Approach Paired With Tandem Mass Spectrometry}

Justyna A. Dobrowolska Zakaria

Northwestern University Feinberg School of Medicine

Randall J. Bateman

Washington University School of Medicine

Monika Lysakowska

Northwestern University Feinberg School of Medicine

Ammaarah Khatri

Northwestern University Feinberg School of Medicine

Dinorah Jean-Gilles

Merck \& Co., Inc.

Matthew E. Kennedy

Merck \& Co., Inc.

Robert J. Vassar ( $\nabla$ r-vassar@northwestern.edu )

Northwestern University Feinberg School of Medicine

Research Article

Keywords:

Posted Date: February 24th, 2022

DOI: https://doi.org/10.21203/rs.3.rs-1321675/v1

License: (c) (i) This work is licensed under a Creative Commons Attribution 4.0 International License.

Read Full License 


\section{Abstract}

Evidence suggests that $\beta$-secretase (BACE1), which cleaves Amyloid Precursor Protein (APP) to form SAPP $\beta$ and $A \beta$, is elevated in AD brains and biofluids and, thus, BACE1 is a therapeutic target for this devastating disease. The direct product of BACE1 cleavage of APP, SAPP $\beta$, serves as a surrogate marker of BACE1 activity in the central nervous system (CNS). This biomarker could be utilized to better understand normal APP processing, aberrant processing in the disease setting, and modulations to processing during therapeutic intervention. In this paper, we present a method for measuring the metabolisms of SAPP $\beta$ and another APP proteolytic product, SAPPa, in vivo in humans using stable isotope labeling kinetics (SILK), paired with immunoprecipitation and liquid chromatography/tandem mass spectrometry (LC/tandem MS). The method presented herein is robust, reproducible, and precise, and allows for the study of these analytes by taking into account their full dynamic potential as opposed to the traditional methods of absolute concentration quantitation that only provide a static view of a dynamic system. A study of in vivo CSF SAPP $\beta$ and SAPPa kinetics using these methods could reveal novel insights into pathophysiological mechanisms of AD, such as increased BACE1 processing of APP.

\section{Introduction}

Alzheimer's disease (AD) is the most common neurodegenerative disease in the elderly. The brains of patients with $A D$ are characterized by an abundance of amyloid- $\beta(A \beta)$ plaques and neurofibrillary tangles. Studies in dominantly inherited $A D$ (DIAD) have indicated that $A \beta$ plaques start accumulating in the brain 15 to 20 years prior to cognitive symptom onset ${ }^{1}$. These plaques are comprised of $A \beta$ proteins, particularly $A \beta_{1-42}$, that have been cleaved from the Type-I transmembrane protein Amyloid Precursor Protein (APP) in a concerted manner first by $\beta$-secretase and second by $\gamma$-secretase. During the initial cleavage of APP by $\beta$-secretase ${ }^{2-5}$ (BACE1), a soluble APP- $\beta$ (SAPP $\beta$ ) protein fragment is produced. Alternatively, APP may be cleaved by a-secretase, releasing SAPPa, which is 16 amino acids longer than SAPP $\beta$. The a-secretase cleavage precludes the formation of the toxic $A \beta_{1-42}$ and, as a result, is generally considered the protective APP processing pathway. Absolute levels of brain BACE1 protein, mRNA, and BACE1 activity in the cerebrospinal fluid (CSF) have been reported to be increased in studies of $A D^{6-9}$ and we have previously shown an increase in the absolute concentration of SAPP $\beta /$ SAPPa in the CSF of humans with amyloid brain deposits, indicating a shift toward the $\beta$-secretase processing pathway of APP in the setting of amyloidosis ${ }^{10}$. As a result, BACE1 became an attractive therapeutic target for $A D$ and there have been several BACE1 inhibitor clinical trials on patients with $A D$ in the last decade ${ }^{11-13}$. As SAPP $\beta$ is the direct product of BACE1 cleavage of APP, it serves as an important surrogate marker of BACE1 activity in the brain under normal conditions, as well as being a marker that can be used to determine the effectiveness of therapeutic intervention. Most studies to date have focused on measuring $A \beta$ levels when studying $A D$ or BACE1 inhibitors, although $A \beta$ is a less proximal product of BACE1 activity. Additionally, most studies have measured absolute concentrations of proteins using methods such as ELISA or Western blotting. In our current study, we have developed a method to measure the metabolism of human SAPP $\beta$ and SAPPa in vivo using stable isotope labeling kinetics (SILK) in an effort to use 
measures of protein metabolism to understand pathophysiologic changes in AD compared to non-AD participants, as well as consider potential differences in kinetics of these proteins within the heterogenous pool of AD patients.

\section{Results}

\section{Sample Preparation/Immunoprecipitation}

The sample preparation for the human CSF was based on a method we previously developed for measuring SAPP $\beta$ and SAPPa in rhesus monkey $\mathrm{CSF}^{14}$ using serial immunoprecipitation with Mrk61 and W0-2 antibodies, covalently bound to CNBr Sepharose 4B beads (GE Healthcare) (courtesy of Merck Research Laboratories). Mrk61 recognizes the KM-neo-epitope of sAPP $\beta$ which is exposed after APP is cleaved by BACE1 (Fig. 1A). The generation and specificity characterization were described previously ${ }^{15,16}$. W0-2 antibody (EMD Millipore) has an epitope several amino acids C-terminal to the BACE1 cleavage of APP shown in Fig. 1A. Both antibody epitopes are present in all three APP isoforms: $A P P_{695}, A P_{751}$ and $A P P_{770}$. APP 695 is the predominant $A P P$ in neuronal cells and would be the largest contributor to the pools of SAPP $\beta$ and sAPPa measured in human CSF. There were important modifications to the previously described protocol ${ }^{14}$ that were incorporated in order to allow for optimal measurements in the human samples. Firstly, the addition of Tween20 prior to immunoprecipitation improved the amount of protein that was isolated and available for digestion (Supplementary Fig. S1A). These tests were run on $\mathrm{H}_{4}-\mathrm{APP}_{\mathrm{wt}}$ cell culture media samples using the Mrk61 IP as this IP had lower recovery of protein than the W0-2 IP. Various volumes of bead slurry were tested and $65 \mu \mathrm{L}$ Mrk61 for CSF and $100 \mu \mathrm{L}$ Mrk61 for media standards produced optimal IP efficiency (data for media shown in Supplementary Fig. S1B). W0-2 IP utilized 30 $\mathrm{LL}$ that quantitatively purified sAPPa. Representative Western Blots show the ability of the antibody beads to clear the CSF of the protein of interest after an overnight (approximately $22 \mathrm{~h}$ ) incubation (Fig. 1B, C). Final immunoprecipitation efficiency was measured quantitatively using the MSD 96-well Multi-Spot sAPPa/sAPP $\beta$ Assay. Aliquots were taken from the original CSF vial, as well as from all the steps during the immunoprecipitation protocol and assayed for SAPP $\beta$ and SAPPa. We measured an effect of $2-7 \%$ protein loss for both proteins during handling (transferring sample from one tube to another and measuring protein concentrations from both aliquots), which could reflect protein adhering to the walls of the aliquot's microcentrifuge tubes or to the walls of the pipet tip. Intraplate CVs for the assay were $<5.5 \%$. Interplate CVs were $<4 \%$ (Fig. 1D). The MSD assay showed that approximately $15 \%$ of starting SAPP $\beta$ in the CSF remained after Mrk61 immunoprecipitation (Fig. 1D). When SAPP $\beta$ was quantified in the supernatant from the subsequent W0-2 IP, $14 \%$ of it remained. This is within the CV range and indicates no significant interaction between W0-2 beads and SAPP 3 . Supernatant after Mrk61 immunoprecipitation had approximately $93 \%$ of pre-Mrk61immunoprecipitation sAPPa (Fig. 1D). However, this loss of 7\% is likely an effect of protein loss during extra handling steps as we had measured previously, and not due to any interaction between Mrk61 and SAPPa. Previous, more extensive, validation studies have demonstrated that there was no such crossreaction ${ }^{15}$. 
We tested on-bead digest vs off-bead (utilizing Formic Acid (FA) to elute proteins off the beads prior to digestion ${ }^{14}$ ) but had higher recovery with on-bead digest (Supplementary Fig. S1C). These tests were done on the Mrk61 IP samples as the recovery is generally poorer for Mrk61 IP than for W02 IP. The SAPP $\beta$ recovered after FA elution was only a fraction of the protein on beads after Mrk61 IP. Tryptic digests of eluted samples and on-bead samples indicated an efficient digest under both circumstances, with only the starting material before the digestion step favoring the on-bead digest. We additionally tested two proteases, mass spectrometry grade Trypsin Gold (Promega) vs. MS grade LysN protease (Pierce) as in Dobrowolska et al. (2014b), but Trypsin Gold gave greater sequence coverage and produced the optimal peptides for Selected Reaction Monitoring (SRM). The tryptic peptide VESLEQEAANER was chosen due to it resulting in the highest signal intensity (Supplementary Fig. S1D). From the LysN peptides, the highest signal intensity was produced when measuring the peptide KYLETPGDENEHAHFQ, but its signal intensity was still below the LLOQ of $1 \mathrm{E} 5$ for the ${ }^{12} \mathrm{C}$-peptide. Trypsin versus LysN experiments were performed on the TSQ Quantum Ultra.

\section{LC/MS SRM Quantitation}

The peptides ( $\left({ }^{12} \mathrm{C}-\mathrm{VESLEQEAANER},{ }^{13} \mathrm{C}\right.$-VESLEQEAANER, and [U: $\left.\left.{ }^{15} \mathrm{~N}\right] \mathrm{VESLEQEAANER}\right)$ eluted and ionized well for both SAPP $\beta$ and SAPPa samples. The endogenous and labeled peptides co-eluted, as expected, and had a retention time (RT) of approximately $13.5 \mathrm{~min}$ along the gradient described in the methods (Fig. 2A). Occasional variability in the analytical columns resulted in insignificant tailing on certain columns. Analytical columns that resulted in significant tailing were not used for sample runs. The $b$ - and $y$-ions (daughter ions) monitored and used in the quantitation also show as a co-eluting stack (Fig. 2B, C, D).

The two sets of standard curves generated from H4-APP ${ }_{\text {wt }}$ conditioned media for both SAPP $\beta$ and SAPPa showed linearity $\left(R^{2}=0.9996\right.$ and $R^{2}=1$, respectively, for standard curve \#1 and $R^{2}=0.9999$ and $R^{2}=1$, respectively, for standard curve \#2 (Supplementary Fig. S2, Supplementary Table S1)). For curve \#1 the slopes were around 0.84 and 0.77 , respectively, and for curve \#2 the slopes were around 0.71 and 0.68 , respectively, indicating that there was not $100 \%$ label in the cultures and experimental readouts were lower than theoretical values (Supplementary Fig. S2, Supplementary Table S1). The first set of standards had slightly better label incorporation and was initially chosen to run alongside each CSF set.

The ${ }^{13} \mathrm{C} /{ }^{12} \mathrm{C}$-peptide ratio was calculated at each CSF time-point for the SAPP $\beta$ and sAPPa samples and a raw kinetic curve was generated over the $36 \mathrm{~h}$ time-course (Fig. 3). $\mathrm{H}_{4}-\mathrm{APP}_{\mathrm{wt}}$ media isotopic enrichment standards were used to generate a standard curve and the raw kinetic values were normalized to the standard curves and plotted against time (Supplementary Fig. S2E). Samples were run in duplicate or triplicate and repeat injections indicated a high level of instrumental precision. In the representative subject's kinetic curves the max Standard Error of the Mean (SEM) at any time-point was $0.06 \%$ in the raw dataset and $0.08 \%$ in the normalized dataset. The majority of data points have an SEM of $</=0.05 \%$ for both SAPP $\beta$ and SAPPa in experiments run continuously over a three-day period, with repeat injections occurring $\sim 48 \mathrm{~h}$ apart. 
Additionally, we tested the reproducibility of this approach on the same samples after a much longer interval. CSF time-courses for another human subject were run on the system 10 months apart so that the analytical and trap columns used in each instance were from different lots. Solvents were different, the API optics of the QqQ had been cleaned multiple times in between the two runs, and the LC/MS system had undergone preventative maintenance in the interim. Even with an abundance of variable changes and with samples being frozen at $-80^{\circ} \mathrm{C}$ for a duration of 10 months, the raw and normalized (to standard curve \#1) kinetic curves generated for both SAPP $\beta$ and SAPPa were nearly identical (Supplementary Fig. S3). The mean CV for the SAPP $\beta$ kinetic curve data points was $6.0 \%$ if all hours were included (Supplementary Table S2). Baseline hours ( $\mathrm{h7}$ and 8) had higher CVs, as their means are near zero, thus very minute differences in standard deviation will result in naturally higher $\mathrm{CVs}$. If excluding baseline $\mathrm{h} 7$ and 8 , the average CVs for the SAPP $\beta$ kinetic curve data points was $3.1 \%$. The mean CVs for the sAPPa kinetic curve data points were $1.9 \%$ if all hours were included and $1.8 \%$ when baseline hours 7 and 8 were excluded.

The CSF used for this study had previously been immunoprecipitated to isolate $A \beta$ for another study using the mid-domain $A \beta$ antibody HJ5.1 ${ }^{17}$. A subset of the subjects' CSF had been processed differently with regard to the $A \beta$ immunoprecipitation, having instead undergone a serial immunoprecipitation with $C$-terminal $A \beta$ specific antibodies, $21 F 12$ and $2 G 3$ to isolate $A \beta_{42}$ and $A \beta_{40}$, respectively ${ }^{18}$. $A$ small fraction of the subjects' CSF had undergone both an HJ5.1 immunoprecipitation and a 21F12/2G3 immunoprecipitation with identical aliquots. We investigated whether the type of immunoprecipitation for $A \beta /$ pre-treatment of CSF would have an impact on SAPP $\beta$ and SAPPa kinetics. The kinetic curves generated for both SAPP $\beta$ and SAPPa with CSF from both types of prior $A \beta$ immunoprecipitations indicate nearly superimposable data points (Supplementary Fig. S4A-B). Mean CV for SAPP $\beta$ data points was $2.4 \%$ (all hours) and 2.2\% (excluding h7 and h8) (Supplementary Table S3). Mean CV for sAPPa data points was $3.7 \%$ (all hours) and $3.2 \%$ (excluding $\mathrm{h} 7$ and $\mathrm{h} 8$ ). As previously noted, the CVs were generally slightly higher ( $\max 4.5 \%$ for SAPP $\beta$ and $13.2 \%$ for SAPPa) at the baseline hours of the kinetic curve where mean values are close to zero and low standard deviations will be reflected with a higher $\mathrm{CV}$ ).

We tested our method on two QqQ models from Thermo Fisher Scientific. The Quantum Ultra was an older generation QqQ used during the initial course of the study, whereas a newer generation TSQ Altis was used during the majority of the study later on. A human CSF time-course previously immunoprecipitated with 21F12/2G3 underwent a serial immunoprecipitation with Mrk61 and W0-2 following the aforementioned protocol. Aliquots from the same sample vials were injected on identical LCs interfaced with either QqQ. Signal intensities on both QqQs were above LLOQ (> $1 \mathrm{E} 5$ for ${ }^{12} \mathrm{C}$ VESLEQEAANER). However, the kinetic curves for both SAPP $\beta$ and SAPPa on the Altis were significantly smoother (Supplementary Fig. S4C-D). The curves for both proteins on the Ultra were erratic and had outlier points as well as significantly higher $\mathrm{CV}$ s, particularly in SAPP $\beta$ replicates (sAPP $\beta$ mean \% CV: $15.2 \%$ (Ultra) vs. $1.1 \%$ (Altis) ; sAPPa mean \% CV: $3.4 \%$ (Ultra) vs. $2.1 \%$ (Altis)). (Supplementary Table S4). The higher CVs were sustained across the full Ultra curves and were not due to the baseline hours as 
hours 7 and 8 were not run in duplicate for this experiment. The data presented in this manuscript, unless otherwise noted, was generated on the Altis.

\section{Absolute Concentration Measurements using Internal Standards and LC/MS}

We measured absolute concentrations of SAPP $\beta$ and SAPPa in CSF samples by addition of ${ }^{15} \mathrm{~N}$ internal standards into CSF prior to immunoprecipitation. $75 \mathrm{ng}$ of [U: $\left.{ }^{15} \mathrm{~N}\right]-\mathrm{sAPP} \beta$ was spiked into each timepoint of CSF from Subject \#1 prior to Mrk61 IP and $75 \mathrm{ng}$ of [U: $\left.{ }^{15} \mathrm{~N}\right]$ - sAPPa was spiked into the supernatant directly following Mrk61 IP and immediately prior to W0-2 immunoprecipitation. The mean concentration of sAPPß was $2907 \mathrm{ng} / \mathrm{mL}$ (min: $2354 \mathrm{ng} / \mathrm{mL}$; max: $3408 \mathrm{ng} / \mathrm{mL}$; CV: 11\%) and the mean concentration of sAPPa was 3110 ng/mL (min: 2309 ng/mL; max: 3689 ng/mL; CV: 12\%) (Supplementary Table S5). The mean ratio of sAPPa/sAPPß was 1.09 (min: 0.85; max: 1.51 ; CV: 18\%). This observed variability in SAPP $\beta$ and sAPPa concentrations across a $36 \mathrm{~h}$ time-course is expected given the physiological diurnal fluctuations of sAPP proteins we reported previously ${ }^{14}$.

\section{Discussion}

Herein we present a novel method utilizing immunoprecipitation and LC-tandem MS to independently measure the in vivo SAPP $\beta$ and SAPPa kinetics in the CSF of humans who had underwent a SILK study. To date, reported measures of SAPP proteins in the CSF of humans have been absolute concentrations of static values using traditional methods such as ELISA. Our approach differs from these methods as a kinetic curve allows measurement of the dynamic nature of proteins in a living system, such as the production and clearance (turnover) of a protein, in order to better understand both the physiological mechanisms of the protein, as well as pathophysiological changes in a disease setting.

Our method allows for the quantitation of SAPP $\beta$ and SAPPa using highly specific antibodies to initially isolate each protein of interest and then the highly sensitive LC/tandem MS approach is used to quantify a common mid-domain APP peptide which not only occurs in both proteins but is found in all three APP isoforms from which SAPP $\beta$ and SAPPa may arise after proteolytic processing. The LC/tandem MS method is incredibly robust and reproducible. The precision of the assay $(<3-5 \%)$ is much better than traditional methods (typical \%CV < 20\%) when samples are assayed a year apart and the instrument had undergone maintenance and consumables from varying lots were used. Additionally, we report that absolute concentrations of SAPP 3 and sAPPa can be calculated using internal standards and IP/MS with our approach. Previous studies using various immunoassay techniques (in-home ELISAs, IBL ELISAs and MSD assays) have reported a wide range of concentrations of these proteins in human CSF (SAPP $\beta=$ 50-1600 ng/mL and sAPPa = 35-2200 ng/mL) ${ }^{10,19-21}$. Our reported values are 1.4-1.8-fold higher than the upper end of the range for both analytes. This large range found in past studies, as well as our current reported values being higher, may be attributed to various sources of antibodies and calibrators/standards used between assays, as well as different stocks of antibodies and calibrators 
within the same assay. Concentrations may also vary based on amyloid status of a subject as well as the time of day the CSF was collected ${ }^{10}$.

Our experiments show that the newer-generation QqQ, the TSQ Altis, has significantly better signal-tonoise ratio and thus, kinetic curves generated on the Altis are smoother and not erratic when compared to samples measured on the Quantum Ultra. The Ultra, additionally, had much higher variability on repeat injections. Going forward with future studies, we utilized the Altis, due to its superiority.

We also show that CSF used to measure SAPP analytes may be processed by two different A $\beta$ immunoprecipitation protocols and the results for SAPP kinetics do not vary significantly. We intend to measure the metabolism of SAPP $\beta$ and SAPPa in 100 human subjects whose CSF had previously been immunoprecipitated for $A \beta$ by HJ5.1 (and a small subset by 21F12/2G3) using this reported method and combine these results with historically available $A \beta$ kinetics for these subjects to better understand the APP processing in healthy humans as well as determine important changes in the whole system that occur in the setting of $A D$.

This approach using our method could potentially be used to determine how to dose therapies for individual clinical trial participants, as well as monitor therapeutic effects. In addition, in vivo CSF SAPP $\beta$ and SAPPa kinetics could reveal novel insights into pathophysiological mechanisms of $A D$, such as increased BACE1 processing and potential for decreased a-secretase processing of APP 22 .

This method could further be modified and applied to study the metabolism of non-APP BACE1 substrates, that have been implicated in chronic adverse events, such as cognitive decline, reported in Phase III BACE1 inhibitor clinical trials ${ }^{23-25}$. BACE1 cleavage of neural cell adhesion molecules, L1 and neural cell adhesion protein close homolog of $\mathrm{L} 1(\mathrm{CHL} 1)$, appears to play a role in synaptic plasticity and learning ${ }^{26-27}$. Conditional BACE1 knockout mouse studies reported defects in axonal organization correlated with a reduction in the BACE1-mediated cleavage of $\mathrm{CHL}{ }^{28}$. Another mouse study reported that Seizure protein 6 (SEZ6) maintains normal dendritic spine dynamics and suggests that aberrations to BACE1-mediated cleavage of SEZ6 upon BACE1 inhibition results in alterations to synaptic function ${ }^{29}$. The adverse effects reported in the clinical trials may be a result of over-inhibition of BACE1 that negatively affects the processing of non-APP substrates. Thus, finding a BACE1 dose that doesn't alter the cleavage and turnover of these other substrates to a degree that impairs cognition, but still inhibits the $\beta$-pathway of APP enough to prevent symptoms of $A D$, is paramount.

Lastly, our method could be applied to study the unique setting of AD in the Down syndrome (DS) population. Although the gross pathological hallmarks of AD in DS are similar to late-onset AD (LOAD) and DIAD, there are critically important differences in the genetic mechanisms that result in this disease. In DS, there is an extra copy of chromosome 21 , and, thereby, an additional copy of the APP gene, located on this chromosome ${ }^{30}$. Therefore, an overproduction of APP, and consequently of its cleavage products, is a lifelong process ${ }^{31}$. The additional copy of APP complicates pharmacokinetic and pharmacodynamic analyses when standard static measures of proteins are utilized to study the mechanisms underlying the 
development of AD in this population. Thus, kinetic analyses of SAPP $\beta$ and SAPPa employing our described method may prove to be beneficial when applied to DS.

\section{Methods}

\section{Stable Isotope Labeling by Amino Acids in Cell Culture (SILAC)}

Human $\mathrm{H} 4$ neuroglioma cells stably transfected with human APP 695 (H4-APP wt $_{6}$ courtesy of T.E. Golde, University of Florida, Gainesville) were cultured in Dulbecco's Modified Eagle Medium (DMEM) (Gibco 10\% dialyzed Fetal Bovine Serum (Gibco), penicillin G and streptomycin (Pen-Strep) (Gibco) and Zeocin (Invitrogen). Cells were split into six flasks evenly and cultured till near confluency. After rinsing with $1 \mathrm{X}$ PBS (Gibco), media was switched to the labeled variant. Labeled media was made with SILAC DMEM devoid of L-Lysine, L-Arginine and L-Leucine (Gibco) with Pen-Strep and Zeocin (FBS-free). L-Lysine-HCl for SILAC (Thermo Scientific) and L-Arginine-HCl for SILAC (Thermo Scientific) were added back into the media at the concentrations found in DMEM. Media was split into six equal volumes, and Leucine was added back in at the concentration found in DMEM, with varying percentages of ${ }^{12} \mathrm{C}$-Leucine: L-Leucine for SILAC (Thermo Scientific) and ${ }^{13} \mathrm{C}$-Leucine: L-Leucine ${ }^{13} \mathrm{C}_{6}, 99 \%$ (Cambridge Isotope Laboratories,

Inc.) to make six medias with $\frac{13 \mathrm{C}-\text { Leucine }}{13 \mathrm{C}-\text { Leucine }+12 \mathrm{C}-\text { Leucine }}=0\left(100 \%{ }^{12} \mathrm{C}\right.$-Leucine $), 1.25,2.5,5,10$, and $20 \%$. Cells were cultured in these media for $24 \mathrm{~h}$ after which media was collected, filtered and stored in aliquots at $-80^{\circ} \mathrm{C}$. The data herein is represented as the ratio of the labeled daughter ions to the unlabeled daughter ions $(\mathrm{L} / \mathrm{U})$ so the standards of $0,1.25,2.5,5,10$, and $20 \%$ correspond to $0,1.27,2.56,5.26$, 11.11 , and $25 \%$.

\section{Antibody Bead Conjugation for Immunoprecipitation}

Mrk61, a neo-epitope specific anti-sAPP $\beta$ rabbit monoclonal antibody (courtesy of Merck Research Laboratories), was generated and its specificity was previously characterized ${ }^{15,16}$. The purified antibody was conjugated with CNBr-activated Sepharose 4B beads (GE Healthcare) according to the manufacturer's protocol, reconstituted into a $50 \%$ slurry of PBS containing $0.02 \%$ sodium azide and stored at $4^{\circ} \mathrm{C}$.

Purified W0-2 (courtesy of Merck Research Laboratories) was added to activated CNBr-Sepharose (1\% $\mathrm{w} / \mathrm{w})$ pre-equilibrated in cold coupling buffer $(0.1 \mathrm{M}$ bicarbonate $\mathrm{pH} 7.6,0.5 \mathrm{M} \mathrm{NaCl})$ and incubated for 2 hours with gentle mixing. Upon washing with coupling buffer, unoccupied binding sites were blocked for 2 hours with $1 \mathrm{M}$ ethanolamine, $\mathrm{pH}$ 8.0. Beads were washed with three cycles of $90 \mathrm{~mL}$ cold $0.1 \mathrm{M}$ acetate $\mathrm{pH} 4.0$ and $90 \mathrm{~mL}$ cold $0.1 \mathrm{M}$ tris $\mathrm{pH} 8.0$ to remove uncoupled protein. The beads were further washed and stored $\left(4^{\circ} \mathrm{C}\right)$ in PBS containing $0.02 \%$ sodium azide.

\section{Regulatory Compliance}


The collection of CSF for human studies took place at the Washington University School of Medicine in St. Louis and was approved by the Washington University Human Studies Committee and the General Clinical Research Center Advisory Committee. The study was performed in accordance with guidelines and regulations. All participants completed informed written consent.

\section{CSF Collection and Sample Preparation}

CSF used for this study was banked at the Bateman Laboratory at Washington University and had been previously utilized for other studies. The CSF was initially collected from human participants under the approval of the Washington University Human Studies Committee and the General Clinical Research Center (GCRC) Advisory Committee. Written, informed consent was obtained from all participants prior to

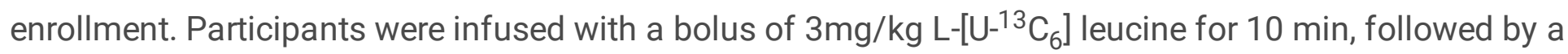
constant infusion $(2 \mathrm{mg} / \mathrm{kg} / \mathrm{h}$ ) for $8 \mathrm{~h} 50 \mathrm{~min}$. CSF was collected hourly for 36 hours (first CSF draw occurred between 8-9:30AM) through an indwelling intrathecal lumbar catheter placed between the L3 and L4 interspace or between the L4 and L5 interspace. Upon collection, CSF was immediately frozen and stored at $-80^{\circ} \mathrm{C}$ as previously described ${ }^{32}$. Prior to this study, aliquots of $1 \mathrm{~mL}$ CSF from each collection time-point were thawed and immunoprecipitated at $4^{\circ} \mathrm{C}$ with HJ5.1 antibody (Washington University, St. Louis, MO) to isolate $A \beta$ as described in previously ${ }^{17}$. CSF time-courses from a small subset of subjects underwent a serial immunoprecipitation with 21F12/2G3 antibodies in lieu of HJ5.1 immunoprecipitation as described previously ${ }^{18}$.

The supernatant, now devoid of $A \beta$, was removed and was frozen and stored at $-80^{\circ} \mathrm{C}$ until this study was initiated. To isolate APP proteolytic products, CSF from a baseline sample of hour 7 during the timecourse, as well as the even hours between hours 8-36 of the study, were thawed at $4^{\circ} \mathrm{C} .500 \mu \mathrm{L}$ of $\mathrm{CSF}$ was used for the study and $500 \mu \mathrm{L}$ of $1 \mathrm{X}$ Phosphate Buffered Saline (PBS) was added to the CSF to bring the starting volume to $1000 \mu \mathrm{L}$. A set of $\mathrm{H}_{4}-\mathrm{APP}_{\mathrm{wt}}$ media isotopic enrichment standards $(1000 \mu \mathrm{L}$ each of MFL $0,1.25,2.5,5,10$, and $20 \%{ }^{13} \mathrm{C}_{6}$-Leucine) was processed alongside each set of CSF. To each aliquot Tween20 (Sigma Aldrich) was added such that there was 0.05\% Tween20 in the final volume to be immunoprecipitated. Protease inhibitors were added for a final sample concentration of $183 \mathrm{ng}$ Leupeptin/mL (Sigma-Aldrich) and 365 ng Aprotinin/mL (Sigma-Aldrich). 75 ng of [U: ${ }^{15} \mathrm{~N}$-sAPPß (Biologics Corp) was spiked into each sample. CSF was serially immunoprecipitated to separately isolate SAPP $\beta$ and sAPPa in a method modified from the previously reported protocol ${ }^{14}$ in which the following antibodies were previously used to isolate the two proteins from rhesus monkey CSF. Samples were incubated with Mrk61 antibody bead slurry (65 $\mu \mathrm{L}$ for CSF and $100 \mu \mathrm{L}$ for media standards) and rotated overnight (approximately $22 \mathrm{~h}$ ) at $4^{\circ} \mathrm{C}$. Supernatants were collected the following day and $75 \mathrm{ng}$ of [U: ${ }^{15} \mathrm{~N}$ sAPPa (Biologics Corp) was spiked into each sample. Samples were subsequently incubated with $30 \mu \mathrm{L}$ W0-2 antibody bead slurry and rotated overnight (approximately $22 \mathrm{~h}$ ) at $4^{\circ} \mathrm{C}$. W0-2 antibody is often utilized as an $A \beta$ peptide detection antibody, however, we took advantage of the antibody epitope (aa 5-8 of $\mathrm{Ab})^{33}$ being located at the N-terminus of the sAPPa sequence and thus having the capability of also pulling down sAPPa. Post-immunoprecipitation Mrk61 beads bound to sAPP 3 , and subsequently post- 
immunoprecipitation W0-2 beads bound to SAPPa, underwent on-bead tryptic digest with Trypsin Gold (Promega) for approximately $20 \mathrm{~h}$ at $37^{\circ} \mathrm{C}$ while shaking. Resultant peptides were desalted using $\mathrm{C} 18$ spin columns (Pierce) and eluted with $70 \%$ acetonitrile (ACN). Eluents were dried in a rotary evaporator (Savant SPD111) at room temperature and resuspended in $20 \mu \mathrm{L} 0.2 \%$ trifluoracetic acid (TFA) for all Mrk61 samples as well as for the W0-2 CSF. W0-2 media standards was resuspended in $30 \mu \mathrm{L} 0.2 \%$ trifluoracetic acid (TFA). For experiments utilizing LysN (Pierce), 5ng MS-grade LysN was added in lieu of Trypsin.

\section{Protein Electrophoresis/Western Blotting}

CSF samples prior to immunoprecipitation and the supernatant, following immunoprecipitation with either Mrk61 or W0-2, was prepared for gel electrophoresis/western blotting by removing $2 \%$ of the full sample. This sample was added to NuPage LDS sample buffer (Invitrogen) with $\beta$-mercaptoethanol (Sigma Aldrich) and boiled for $10 \mathrm{~min}$ at $95^{\circ} \mathrm{C}$. Equivalent volumes of sample were loaded into wells of a NuPage 4-12\% Bis-Tris gel (Invitrogen) and MOPS buffer (Invitrogen) was used. The gel was run at 200V. The proteins were transferred onto a PVDF membrane (Fisher Scientific) by a wet transfer utilizing $1 \mathrm{X}$ Tris-Glycine Transfer Buffer with $20 \%$ Methanol. Transfers occurred overnight ( $18 \mathrm{~h})$ at $4^{\circ} \mathrm{C}$. PVDF membranes were blocked with Superblock (Pierce) in TBS for $1 \mathrm{~h}$ at room temperature (RT). Dilutions of primary antibodies Mrk61 (Merck) ${ }^{15,16}$ and 6E10 (BioLegend) ${ }^{34}$ were made in 10\% PBS blocking buffer (Pierce) in PBS-T and incubated with membranes at RT for $1 \mathrm{~h}$. Membranes were rinsed in PBS-T. Secondary antibodies (goat anti-rabbit IgG antibody and horse anti-mouse IgG antibody, respectively) (Vector Laboratories) were diluted in 10\% PBS blocking buffer in PBS-T and incubated with membranes for $1 \mathrm{~h}$ at RT, followed by PBS-T rinses. For Mrk61 blots, SuperSignal West Femto Maximum Sensitivity Substrate (Thermo Fisher) was used to develop. For 6E10 blots, standard SuperSignal Substrate (Thermo Fisher) was used for development. Blots were developed using the FluorChem R Imaging System (ProteinSimple).

\section{MesoScale Discovery (MSD) Assay}

Prior to and after immunoprecipitation the CSF samples and post-IP supernatant (following immunoprecipitation with either Mrk61 or W0-2) were prepared for protein concentration analysis utilizing a 96-well Multi-Spot sAPPa/sAPP $\beta$ Assay (MSD). A plate was blocked with Blocker A solution (MSD) for $1.5 \mathrm{~h}$ at RT with shaking and washed with $1 \mathrm{X}$ Tris buffer. Samples were diluted 35 -fold in Diluent 35 (MSD). Samples and calibrators were loaded onto the plate at a $25 \mu \mathrm{L}$ volume and incubated at RT with shaking for $1 \mathrm{~h}$. Plates were washed with $1 \mathrm{X}$ Tris buffer and detection antibody from the kit was added per MSD protocol instructions and incubated at RT with shaking for $1 \mathrm{~h}$. Plates were washed again with $1 \mathrm{X}$ Tris buffer. 1X Read Buffer was added to the wells and the plate was incubated without shaking for 10 min and was read using a Sector S600 imager (MSD).

\section{Liquid chromatography/Mass Spectrometry}

Samples were run on the nano liquid chromatography (nanoLC)-triple quadrupole (QqQ) mass spectrometer: UltiMate 3000 RS nanoLC-TSQ Altis (Thermo Fisher Scientific). An autosampler was 
interfaced with the liquid chromatography system and samples were kept chilled at $8^{\circ} \mathrm{C}$ on the autosampler until injection. The TSQ Altis was equipped with a New Objective (Littleton, MA) nanospray ionization (NSI) source. Some experiments were performed on a Thermo Quantum Ultra QqQ (an older generation instrument) as a comparative test of the two instruments. In this case, the LC and autosampler systems and all other parameters were kept constant. Volume injected for CSF sample aliquots was $4 \mu \mathrm{L}$. For media isotopic enrichment standards, injection volumes were as follows: $4 \mu \mathrm{L}$ for sAPP $\beta$ standards and $2 \mu \mathrm{L}$ for sAPPa standards. Samples were injected in duplicate (and occasionally in triplicate) onto a New Objective Picochip analytical column that had a $105 \mathrm{~mm}$ length bed, an internal diameter (ID) of $75 \mu \mathrm{m}$, tip size of $15 \mu \mathrm{m}$ and packed with H080 ProntoSIL C18-Aq 3 $\mu \mathrm{m}$ 200A media. The $\mathrm{LC}$ was run on a $300 \mathrm{~nL} / \mathrm{min}$ flowrate over the course of a 30 -minute gradient. Mobile Phase $\mathrm{A}$ was $0.1 \%$ Formic Acid (FA) and mobile phase B was $0.1 \%$ FA, $80 \%$ ACN/20\% water. The gradient was $1 \%$ B for the initial $5 \mathrm{~min}$ and then increased from $1 \%$ B to $95 \%$ B from $5-15 \mathrm{~min}$. It remained at $95 \%$ B from $15-23$ min and then dropped from $95 \%$ B to $1 \%$ B between $23-24$ min. The system re-equilibrated at $1 \%$ B from 24-30 min.

The SRM experiment took place between 10-18 min and was run with the instrument in positive polarity. Cycle time was $0.7 \mathrm{sec}$ and the collision pressure gas setting was $1.5 \mathrm{mTorr}$. Q1 and Q3 resolutions were both set at $0.7 \mathrm{FWHM}$. The RF Lens setting was $90 \mathrm{~V}$. The parent ions monitored were $\mathrm{m} / \mathrm{z}=687.828++$ $\left({ }^{12} \mathrm{C}\right.$-VESLEQEAANER) and $690.838++\left({ }^{13} \mathrm{C}\right.$-VESLEQEAANER) (Supplementary Table S6). The daughter (product) ions monitored for ${ }^{12} \mathrm{C}$-VESLEQEAANER were $\mathrm{b} 3=316.1503+\mathrm{y} 10=1146.5386+\mathrm{y} 9=$ $1059.5065+$, y $8=946.4225+, y 7=817.3799+, y 6=689.3213+$, and $y 5=560.2787+$. The daughter (product) ions monitored for ${ }^{13} \mathrm{C}$-VESLEQEAANER were $\mathrm{b} 3=316.1503+\mathrm{y} 10=1152.5587+\mathrm{y} 9=$ $1065.5267+, \mathrm{y} 8=946.4225+, \mathrm{y} 7=817.3799+, \mathrm{y} 6=689.3213+$, and $\mathrm{y} 5=560.2787+$.

\section{Data Analyses}

Raw data generated through LC/tandem MS was imported into Skyline 21.1.0.146 (Univ. of Washington), normalized to the media isotopic enrichment standards and analyzed within the program. Seven b-and yions were used for quantitation from the ${ }^{12} \mathrm{C}$-VESLEQEAANER peptide, seven corresponding $b$ - and $y$-ions were used for the ${ }^{13} \mathrm{C}$-VESLEQEAANER peptide, and 7 corresponding ions were used for $\left[U:{ }^{15} \mathrm{~N}\right]$ VESLEQEAANER. The ratio of the labeled $\left({ }^{13} \mathrm{C}\right)$ daughter ions to the $\left({ }^{12} \mathrm{C}\right)$ unlabeled daughter ions $(L / U)$ was calculated at each data point along the time-course. The median of replicates was quantified and used to generate a raw kinetic curve. Each data point was also normalized to the corresponding standard curve that was run in parallel with the CSF time-course and normalized time-courses were also plotted. Absolute concentrations were measured by taking the sum of ${ }^{12} \mathrm{C}$ and ${ }^{13} \mathrm{C}$ peak areas and normalizing to $\left[\mathrm{U}:{ }^{15} \mathrm{~N}\right]$ peptide peak area. Raw data, as well as normalized results, were exported into custom-designed Excel (Microsoft) sheets for further analyses. Standard error of the mean (SEM) or standard deviation (Stdev) were calculated for replicates. Coefficient of variation (CV (\%)) was calculated to determine precision when comparisons were made. 


\section{Declarations}

\section{Acknowledgments}

This work was funded by a Mechanisms of Aging and Dementia NIA Training Grant T32 AG20506, the Northwestern University Clinical and Translational Sciences Institute Pilot Award UL1TR001422, through a grant from an Anonymous Foundation, and Brightfocus Foundation ADR grant A2019520S, and the NIH R01NS065667. Todd Golde (University of Florida) generously provided the H4-APP ${ }_{w t}$ cell line used to create the isotopic enrichment standards. Merck Research Laboratories provided continuous Mrk61 and W0-2 antibody bead support throughout this study. The Northwestern University Proteomics Core helped with generating the Quantum Ultra data. We are especially grateful to the SILK study participants for selflessly donating their time and CSF samples for this project.

\section{Author contributions}

J.A.D.Z., R.J.V. and R.J.B. conceived and designed this research study. J.A.D.Z., M.L. and A.K. performed immunoprecipitation experiments. J.A.D.Z. performed all mass spectrometry, electrophoresis/immunoblot, MSD, and SILAC experiments and all subsequent data analyses. D. J.-G. developed the W0-2 antibody coupling method and qualified the resin for purification. M.E.K. supplied the W0-2 and Mrk61 antibody beads. R.J.V. supervised the work. J.A.D.Z. prepared the figures and wrote the manuscript. R.J.V, R.J.B and M.E.K. provided intellectual contributions and critical revisions to the manuscript. J.A.D.Z., R.J.V. and R.J.B. obtained funding. R.J.V. provided the Thermo nanoLC and Altis mass spectrometer. R.J.B provided the CSF samples. All authors read and approved the final manuscript.

\section{Data Availability statement}

The proteomics datasets generated and/or analyzed during the current study are available in the PeptideAtlas - PASSEL repository, http://www.peptideatlas.org/PASS/PASS01740. All other data generated and analyzed during the current study are available from the corresponding author on reasonable request.

\section{Additional Information}

Competing Interests: The authors declare the following competing interests: J. A. Dobrowolska Zakaria has previously received royalty income based on technology (stable isotope labeling kinetics) licensed by Washington University to C2N Diagnostics. M. E. Kennedy and D. Jean-Gilles are employed by Merck \& Co., Inc. and provided antibodies for this study. R. J. Bateman co-founded C2N Diagnostics. Washington University and R. J. Bateman have equity ownership interest in C2N Diagnostics and receive royalty income based on technology (stable isotope labeling kinetics and blood plasma assay) licensed by Washington University to $\mathrm{C} 2 \mathrm{~N}$ Diagnostics. He receives income from C2N Diagnostics for serving on the scientific advisory board. He consulted for Roche, Genentech, AbbVie, Pfizer, Boehringer-Ingelheim, and Merck. R. Vassar serves on the Scientific Advisory Board of Alector and owns Alector stock. Northwestern 
University and R. Vassar receive royalty income from licensing of the 5XFAD mouse. M. Lysakowska and A. Khatri declare no potential conflict of interest.

\section{References}

1. Bateman, R. J. et al. Clinical and biomarker changes in dominantly inherited Alzheimer's disease. $N$. Engl. J. Med. 367, 795-804 (2012).

2. Vassar, R. et al. Beta-secretase cleavage of Alzheimer's amyloid precursor protein by the transmembrane aspartic protease BACE. Science<bvertical-align:super;></bvertical-align:super;>286, 735-741 (1999).

3. Hussain, I. et al. Identification of a novel aspartic protease (Asp 2) as beta-secretase. Mol. Cell Neurosci. 14, 419-427 (1999).

4. Sinha, S. et al. Purification and cloning of amyloid precursor protein beta-secretase from human brain. Nature 402, 537-540 (1999).

5. Yan, R. et al. (1999) Membrane-anchored aspartyl protease with Alzheimer's disease beta-secretase activity. Nature<bvertical-align:super;>402</bvertical-align:super;>, 533-537 (1999).

6. Fukumoto, H., Cheung, B. S., Hyman, B. T. \& Irizarry, M. C. Beta-secretase protein and activity are increased in the neocortex in Alzheimer disease. Arch. Neurol. 59, 1381-1389 (2002).

7. Holsinger, R. M., Lee, J. S., Boyd, A., Masters, C. L. \& Collins, S. J. CSF BACE1 activity is increased in CJD and Alzheimer disease versus [corrected] other dementias. Neurology 67, 710-712 (2006).

8. Yang, L. B. et al. Elevated beta-secretase expression and enzymatic activity detected in sporadic Alzheimer disease. Nat. Med. 9, 3-4 (2003).

9. Coulson, D. T. et al. BACE1 mRNA expression in Alzheimer's disease postmortem brain tissue. J. Alzheimers Dis. 22, 1111-1122 (2010).

10. Dobrowolska, J. A. et al. Diurnal patterns of soluble amyloid precursor protein metabolites in the human central nervous system. PLoS One 9, e89998; 10.1371/journal.pone.0089998 (2014).

11. Vassar, R. (2014) BACE1 inhibitor drugs in clinical trials for Alzheimer's disease. Alzheimers Res. Ther. <bvertical-align:super;>6</bvertical-align:super;>, 89; 10.1186/s13195-014-0089-7 (2014).

12. Hampel, H. et al. The $\beta$-Secretase BACE1 in Alzheimer's Disease. Biol. Psychiatry 89, 745-756 (2021).

13. McDade, E. et al. The case for low-level BACE1 inhibition for the prevention of Alzheimer disease. Nat. Rev. Neurol. 17, 703-714 (2021).

14. Dobrowolska, J. A. et al. CNS amyloid- $\beta$, soluble APP-a and $-\beta$ kinetics during BACE inhibition. J. Neurosci. 34, 8336-8346 (2014).

15. Wu, G., Sankaranarayanan, S., Hsieh, S. H, Simon, A. J. \& Savage, M. J. Decrease in brain soluble amyloid precursor protein $\beta$ (SAPP $\beta$ ) in Alzheimer's disease cortex. J. Neurosci. Res. 89, 822-832 (2011). 
16. Wu, G. et al. Characterization of plasma $\beta$-secretase (BACE1) activity and soluble amyloid precursor proteins as potential biomarkers for Alzheimer's disease. J. Neurosci. Res. 90, 2247-2258 (2012).

17. Patterson, B. W. et al. Age and amyloid effects on human central nervous system amyloid-beta kinetics. Ann. Neurol. 78, 439-453 (2015).

18. Mawuenyega, K. G. et al. Decreased clearance of CNS beta amyloid in Alzheimer's disease. Science 330, 1774 (2010).

19. Zetterberg, H. et al. Elevated cerebrospinal fluid BACE1 activity in incipient Alzheimer disease. Arch. Neurol. 65, 1102-1107 (2008).

20. Mulugeta, E. et al. Cerebrospinal Fluid Levels of sAPPa and SAPP $\beta$ in Lewy Body and Alzheimer's Disease: Clinical and Neurochemical Correlates. Int. J. Alzheimers Dis. <backgroundcolor:\#66FF66;bvertical-align:super;>2011</background-color:\#66FF66;bvertical-align:super;>, 495025; doi: 10.4061/2011/495025 (2011).

21. van Waalwijk van Doorn, L. J. et al. Validation of soluble amyloid- $\beta$ precursor protein assays as diagnostic CSF biomarkers for neurodegenerative diseases. J. Neurochem. 137, 112-121 (2016).

22. Hartl, D. et al. A rare loss-of-function variant of ADAM17 is associated with late-onset familial Alzheimer disease. Mol. Psychiatry 25, 629-639 (2020).

23. Egan, M. F. et al. Randomized Trial of Verubecestat for Prodromal Alzheimer's Disease. N. Engl. J. Med. 380, 1408-1420 (2019).

24. Wessels, A.M. et al. Cognitive outcomes in trials of two BACE inhibitors in Alzheimer's disease. Alzheimers Dement. 16, 1483-1492 (2020).

25. Sperling, R. et al. Findings of Efficacy, Safety, and Biomarker Outcomes of Atabecestat in Preclinical Alzheimer Disease: A Truncated Randomized Phase 2b/3 Clinical Trial. JAMA Neurol. 78, 293-301 (2021).

26. Kuhn, P. H. et al. Secretome protein enrichment identifies physiological BACE1 protease substrates in neurons. EMBO J. 31, 3157-3168 (2012).

27. Zhou, L. et al. The neural cell adhesion molecules $\mathrm{L} 1$ and $\mathrm{CHL} 1$ are cleaved by BACE1 protease in vivo. J. Biol. Chem. 287, 25927-25940 (2012).

28. Ou-Yang, M.-H. et al. Axonal organization defects in the hippocampus of adult conditional BACE1 knockout mice. Sci. Transl. Med. 10, eaao5620; doi: 10.1126/scitranslmed.aao5620 (2018).

29. Zhu, K. et al. Beta-Site Amyloid Precursor Protein Cleaving Enzyme 1 Inhibition Impairs Synaptic Plasticity via Seizure Protein 6. Biol. Psychiatry. 83, 428-437 (2018).

30. Zis, P. \& Strydom, A. Clinical aspects and biomarkers of Alzheimer's disease in Down syndrome. Free Radic. Biol. Med. 114, 3-9 (2018).

31. Wiseman, F. K. et al. A genetic cause of Alzheimer disease: mechanistic insights from Down syndrome. Nat. Rev. Neurosci. 16, 564-574 (2015).

32. Bateman, R. J. et al. Human amyloid- $\beta$ synthesis and clearance rates as measured in cerebrospinal fluid in vivo. Nat. Med. 12, 856-861 (2006). 
33. Ida, N. et al. Analysis of heterogeneous A4 peptides in human cerebrospinal fluid and blood by a newly developed sensitive Western blot assay. J. Biol. Chem. 271, 22908-22914 (1996).

34. Rose, C. et al. New highly sensitive rodent and human tests for soluble amyloid precursor protein alpha quantification: preclinical and clinical applications in Alzheimer's disease. BMC Neurosci. 13, 84; doi: 10.1186/1471-2202-13-84 (2012).

\section{Figures}

\section{Figure 1}

$\mathrm{APP}_{695}$ isoform sequence and Antibody Efficiency. A) Red arrows point to the BACE1 cleavage sites ( $\beta$ site is the predominant cleave location, with the $\beta^{\prime}$ site being a significantly lesser contributor). Green arrows show the $\alpha$-secretase cleavage sites ( $\alpha^{\prime}$ is a significantly lesser contributor). Purple arrows show $\gamma^{-}$ secretase cleavage sites, that, if preceded by BACE1 cleavage at the $\beta$ cleavage site, would result in $A \beta_{38}$, $A \beta_{40}$, or $A \beta_{42}$, respectively. Mrk61 antibody recognizes the neo-epitope of sAPP $\beta$ (-KM bolded red), whereas W0-2 antibody recognizes a short sequence C-terminal to the $\beta$-secretase cleavage location, which is only located in SAPPa or A 3 (bolded green). The APP mid-domain tryptic peptide quantified by MS is shown in bolded blue. It is a peptide common to both SAPP $\beta$ and SAPPa. Panel created with BioRender.com. B) Efficiency of Mrk61 immunoprecipitation showing sAPP $\beta$ in the starting material and in the supernatant of the sample after IP. Original full blot is presented in Supplementary Figure S5. C) Efficiency of W0-2 immunoprecipitation showing SAPPa in the starting material and in the supernatant of the sample after IP. Original full blot is presented in Supplementary Figure S5. D) Percentages of SAPP $\beta$ and SAPPa (from starting material) remaining in supernatants of the serial immunoprecipitation as measured by MSD.

\section{Figure 2}

Parent and daughter ions of peptides measured in the SRM experiment on the TSQ Altis. A) The parent ions monitored were $\mathrm{m} / \mathrm{z}=687.828++\left({ }^{12} \mathrm{C}\right.$-VESLEQEAANER $), 690.838++\left({ }^{13} \mathrm{C}\right.$-VESLEQEAANER $)$ and $696.3032+$ ([U: $\left.{ }^{15} \mathrm{~N}\right]$ VESLEQEAANER) and elute at the identical retention time (RT). B) The transitions for the ${ }^{12} \mathrm{C}$-VESLEQEAANER daughter ions are shown to stack at the same RT. The panel below shows the $\mathrm{m} / \mathrm{z}$ of the $\mathrm{b}$ - and $\mathrm{y}$-ions monitored in blue. C) The transitions for the ${ }^{13} \mathrm{C}$-VESLEQEAANER daughter ions are shown to stack at the same RT. The panel below shows the $m / z$ of the $b$ - and $y$-ions monitored in blue. D) The transitions for the [U: $\left.{ }^{15} \mathrm{~N}\right]$ VESLEQEAANER daughter ions are shown to stack the same RT. The panel below shows the $\mathrm{m} / \mathrm{z}$ of the $b$ - and $y$-ions monitored in blue. 
Subject \#1: sAPP $\beta$ and sAPP $\alpha$ Raw Kinetic Curves

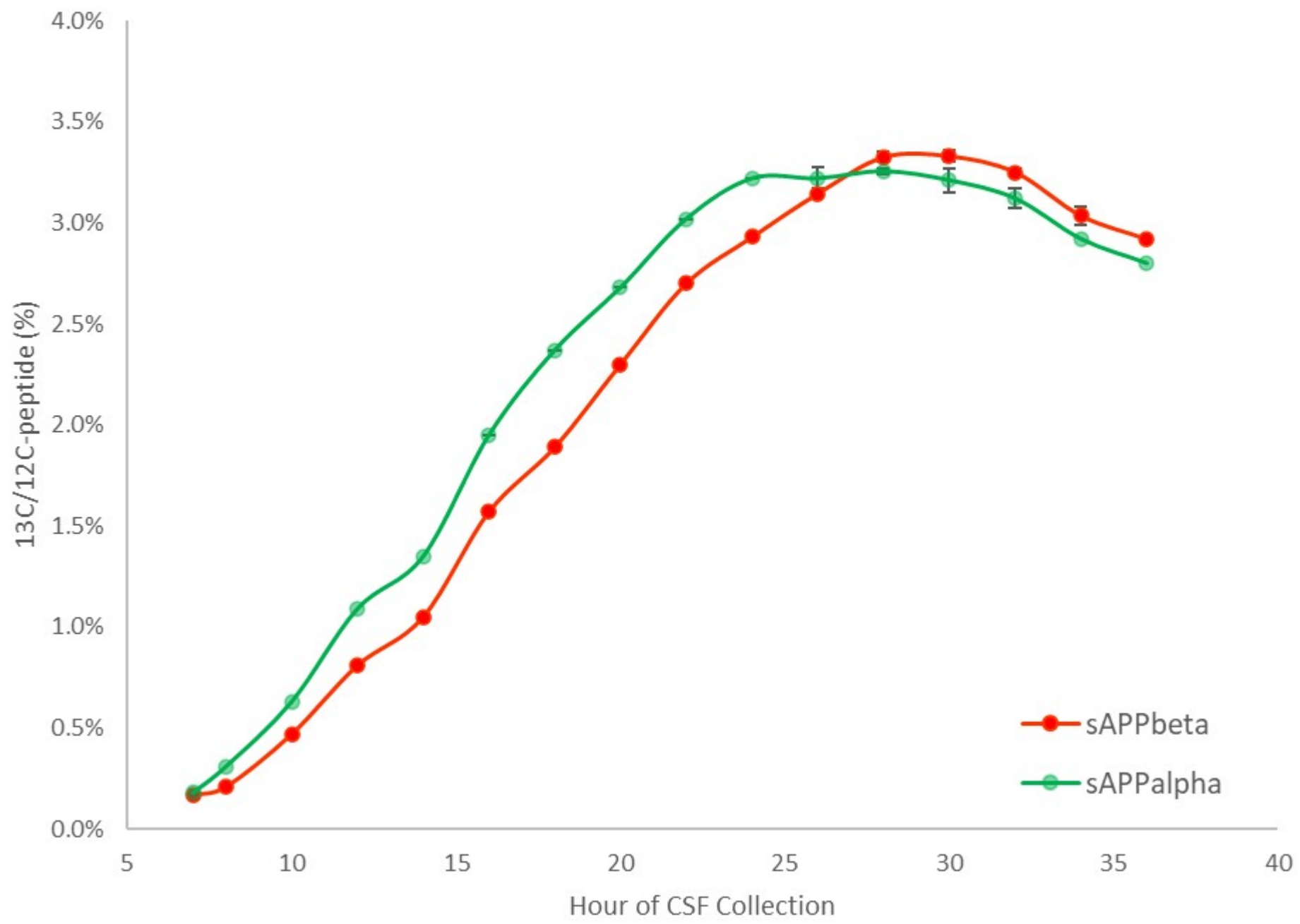

Figure 3

SAPP $\beta$ and sAPPa raw kinetic curves. A sample subject's kinetic curves of sAPP $\beta$ (red) and sAPPa (green) are represented by raw data (Labeled/Unlabeled (\%)) at each data point during the $36 \mathrm{~h}$ CSF collection period. Error bars represent SEM with the data point comprising an average value of duplicate or triplicate injections of a single time-point sample.

\section{Supplementary Files}

This is a list of supplementary files associated with this preprint. Click to download.

- SupplementaryInformationJDZamended.docx 